\title{
Study of the chewing assistance mechanism in powered robotic dentures
}

\author{
Toshitake Araie $^{1}$, Tomozumi Ikeda ${ }^{2}$, Uichi Nishizawa ${ }^{3}$, Akira Kakimoto ${ }^{4}$, Shigeki Toyama ${ }^{5}$ \\ ${ }_{1,2,4}$ Polytechnic University of Japan, Tokyo, Japan \\ ${ }^{3,5}$ Tokyo University of Agriculture and Technology, Tokyo, Japan \\ ${ }^{1}$ Corresponding author \\ E-mail: ${ }^{1}$ araie@uitec.ac.jp, ${ }^{2} i k e d a @ u i t e c . a c . j p,{ }^{3}$ n-uichi@cc.tuat.ac.jp, ${ }^{4}$ kakimoto@uitec.ac.jp, \\ 5toyama@cc.tuat.ac.jp
}

Received 23 July 2018; accepted 5 August 2018

DOI https://doi.org/10.21595/vp.2018.20119

Check for updates

Copyright $(2018$ Toshitake Araie, et al. This is an open access article distributed under the Creative Commons Attribution License, which permits unrestricted use, distribution, and reproduction in any medium, provided the original work is properly cited.

\begin{abstract}
The purpose of this study is to develop powered robotic dentures for elderly people without disabilities to assist them with chewing and swallowing. As the first step, a prototype mechanism was developed to realize the two functions of a molar tooth i.e., crushing and grinding. The results of the measurements of chewing values of the prototype, while showing a decreasing trend in crushing by impact, suggested that chewing is more effective with a combination of rotation and occlusal grooves. Moreover, the use of an ultrasonic motor as a driving actuator in the powered robotic denture was also studied.
\end{abstract}

Keywords: chewing assist, power robotic denture, ultrasonic motor, translational-rotational motor.

\section{Introduction}

The average life expectancy of humans has been increasing over the past few years due to advances in medical care. However, "healthy life expectancy" which refers to the number of years a person is expected to live independently, is shorter than actual life expectancy by about 10 years. Chewing is one of the factors associated with this healthy life expectancy. The ability to chew and swallow declines with age. This may lead to eating and swallowing disorders, dehydration, and low nutrition, thus making it difficult to sustain good health. Since the quality of health and life is strongly associated with the content of food consumed, improving the quality of meals is an important factor towards improving the quality of life.

In the field of dentistry, instruments have been developed for measuring jaw movements and food properties. In order to elucidate the human masticatory movement in terms of engineering, Waseda University has successfully developed a masticatory robot to treat people with jaw movement disorders [1]. In contrast, research on developing equipment for supporting independence in elderly people without disabilities by assisting them with intake of meals has not progressed.

Providing masticatory support robots for elderly people without disorders can lead to a significant increase in their life expectancy and healthy life expectancy. Accordingly, the purpose of this study was to develop powered robotic dentures as a robot realizing a series of functions, including chewing and swallowing movements, to assist elderly people without any disabilities with chewing. The operation of a mechanism to assist with chewing was first studied, and a prototype was designed and developed. The robot in this study is not an exoskeleton-type device as it is designed to be similar to a common denture in which a chewing assistance mechanism has been embedded.

Experiments were conducted to verify the degree of improvement in chewing ability by using the prototype mechanism. Moreover, the results of investigation on the use of ultrasonic motors as driving actuators in powered robotic dentures have also been reported. 


\section{Overview of chewing assistance mechanism}

\subsection{Design of the chewing assistance mechanism}

In general, chewing includes elements of cutting, crushing, and grinding, and the functions vary with the dentition. Among these, this study has focused on the two functions of the molar teeth i.e., crushing by biting into food materials and grinding via friction, and investigated the mechanisms to realize them.

Fig. 1 shows the structure of the assisting mechanism that consists of five parts, including a spring. When a force is applied perpendicular to the occlusal surface, the first part moves downward by rotating along the spiral shape. As it moves downward, the food is ground while it is kept pressed by the force in the spring.

A spring with spring constant of $6 \mathrm{~N} / \mathrm{mm}$ was selected to ensure that the spring can be activated even by elderly people whose occlusal force has decreased. The occlusal surface was designed with parallel grooves to improve the grinding effect. A groove shape with a width of $1 \mathrm{~mm}$ and a depth of $1 \mathrm{~mm}$ was selected, for which favorable results were obtained in a previous study [2].

\subsection{Prototype model of the chewing assistance mechanism}

The modelling was done using 3D CAD software (SOLIDWORKS 2015, Dassault Systèmes) based on the specifications of a chewing assistance mechanism, and the prototype model was built using a Formlabs Form2 3D printer. The 3D printer utilized a stereolithography method to build the model in a spiral shape by orienting the model diagonally and using a stacking pitch of $0.1 \mathrm{~mm}$. Since a 3D printer was used, acrylic material with a compressive strength of $120 \mathrm{MPa}$ was used to build the model. The prototype spiral mechanism model consists of five parts, including the spring, as shown in Figs. 1 and 2. In Fig. 2 we can see, from the left, the upper part, the occlusion part, the spiral guide, and the base part. During assembly, the spring is inserted between the base part and the occlusion part. To verify the chewing assistance mechanism model using experiments, the size of the model is larger than that of an actual tooth. The model's outer diameter is $45 \mathrm{~mm}$, and the rotating occlusion part has an outer diameter of $25 \mathrm{~mm}$ to allow for testing by using peanuts as a chewing material. Fig. 3 shows the prototype model.

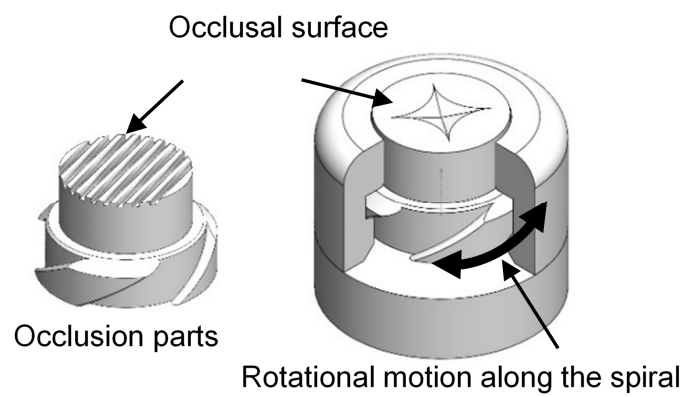

Fig. 1. Structure of the assist mechanism

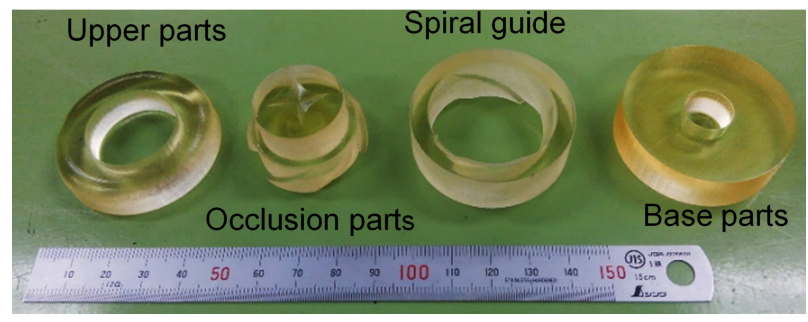

Fig. 2. Component parts

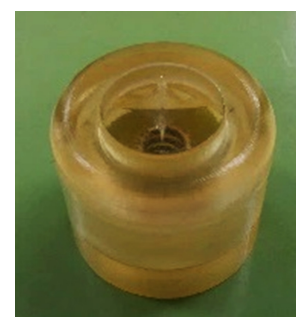

Fig. 3. Prototype model 


\section{Performance of the chewing assistance model}

\subsection{Experimental method}

To verify the chewing performance, four types of mechanisms were developed, as shown in Table 1.

Table 1. Types of mechanisms

\begin{tabular}{|c|c|c|c|c|}
\hline & Rotation & Groove & Spring & Milling method \\
\hline Mechanism A & None & None & None & \multirow{2}{*}{ Crush } \\
\hline Mechanism B & None & Exist & None & \\
\hline Mechanism C & Exist & None & Exist & \multirow{2}{*}{$\begin{array}{l}\text { Compression } \\
\text { friction }\end{array}$} \\
\hline Mechanism D & Exist & Exist & Exist & \\
\hline
\end{tabular}

The conditions for the experiment were established by referring to the Manly method [3] of testing the chewing function, in which the subject is made to chew $3 \mathrm{~g}$ of peanuts 20 times. The masticated food is then sifted through a 10 mesh sieve, its weight measured, and its ratio to the standard amount is determined as the chewing value.

Fig. 4 shows the experimental device. An air cylinder (PBDALS 10×30-M, KOGANEI Corporation, Japan) was used to apply pushing force as an occlusal force, and a load cell (LCN-A-2kN, Kyowa Electronics Company Ltd., Japan) was used for measuring the applied pushing force. The chewing assistance model was placed in a jig for measurements, and the air cylinder was adjusted such that vertical force could be applied to the model. Peanuts were used as a test material similar to the Manly method, and specimens of one half of a peanut kernel around $0.4 \mathrm{~g}$ in weight were selected for use in the experiments. The specimen was placed such that its cross-sectional surface faced the occlusal surface, and a maximum force of $50 \mathrm{~N}$ was applied successively thirty times. The normal occlusal force in healthy individuals with a normal chewing function is about $100 \mathrm{~N}$. However, the value of force applied in the test was $50 \mathrm{~N}$ to account for elderly people. Next, the ground material was sifted through a 10 mesh sieve, and the chewing value was determined by calculating the ratio of the material passing through the sieve with respect to the standard material. The tests were repeated ten times for each condition, and the mean and standard deviation of the chewing values were calculated. These tests were also repeated with pushing forces of $30 \mathrm{~N}$ and $40 \mathrm{~N}$ to investigate the effect of differences in occlusal force on chewing value.

\subsection{Discussion of experimental results}

Table 2 shows the results of the chewing effect verification tests for mechanisms A through D for a pushing force of 30-50 N.

Fig. 5 shows the graph of chewing values for the mechanisms A through D. For a pushing force of $30 \mathrm{~N}$, the mechanisms $\mathrm{C}$ and $\mathrm{D}$ with rotation exhibited values lower than those for mechanisms $\mathrm{A}$ and $\mathrm{B}$ without rotation. For a pushing force of $40 \mathrm{~N}$, all the mechanisms exhibited similar values. For the pushing force of $50 \mathrm{~N}$, mechanism $\mathrm{C}$ exhibited lower values when compared to the other mechanisms. For the $50 \mathrm{~N}$ tests, based on $t$-tests, a significant difference $(p<0.05)$ was observed in the case of mechanism $\mathrm{C}$ when compared to the others.

Upon comparing the results of the tests for $30 \mathrm{~N}$ and $40 \mathrm{~N}$ pushing forces, it was observed that the mechanisms of $\mathrm{A}$ and $\mathrm{B}$ without rotation showed a tendency where the chewing value increased with an increase pushing force. Although the values under $40 \mathrm{~N}$ pushing force for mechanisms $\mathrm{C}$ and $\mathrm{D}$ with rotation were both quite high, no significant difference was observed in comparison to the other mechanisms. One reason for this is because the ratio of the material with sizes smaller than $1.7 \mathrm{~mm}$ passing through the 10 mesh sieve was used to calculate the chewing value, the amount of materials with sizes in the below $1.7 \mathrm{~mm}$ category could not be determined, resulting in an inability to determine the effect of frictional grinding on the chewing 
value. Hence, further tests using sieves lower than 10 mesh are necessary in order to determine the effect of grinding due to friction.

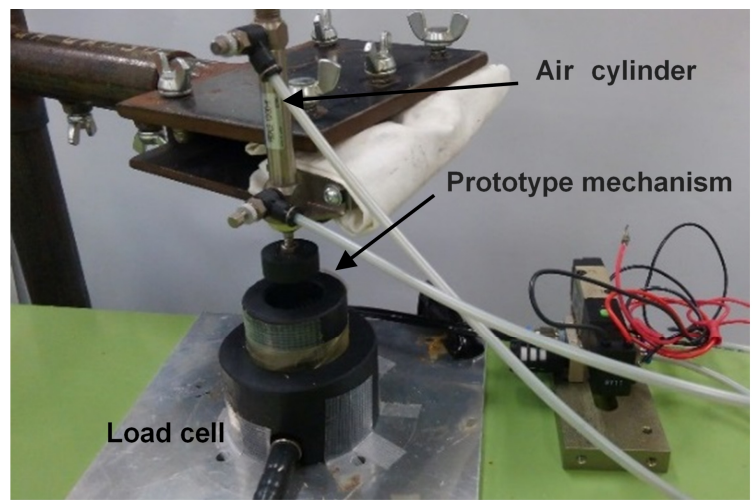

Fig. 4. Experimental device

Table 2. Mean and standard deviation of chewing value

\begin{tabular}{|c|c|c|c|c|c|c|c|c|c|c|c|c|}
\hline & \multicolumn{4}{|c|}{ Pushing force $50 \mathrm{~N}$} & \multicolumn{4}{c|}{ Pushing force $40 \mathrm{~N}$} & \multicolumn{4}{c|}{ Pushing force $30 \mathrm{~N}$} \\
\cline { 2 - 14 } & No rotation & \multicolumn{2}{c|}{ Rotation } & \multicolumn{2}{c|}{ No rotation } & \multicolumn{2}{c|}{ Rotation } & \multicolumn{2}{c|}{ No rotation } & \multicolumn{2}{c|}{ Rotation } \\
\cline { 2 - 13 } & $\begin{array}{c}\text { No } \\
\text { groove }\end{array}$ & Groove & $\begin{array}{c}\text { No } \\
\text { groove }\end{array}$ & Groove & $\begin{array}{c}\text { No } \\
\text { groove }\end{array}$ & Groove & $\begin{array}{c}\text { No } \\
\text { groove }\end{array}$ & Groove & $\begin{array}{c}\text { No } \\
\text { groove }\end{array}$ & Groove & $\begin{array}{c}\text { No } \\
\text { groove }\end{array}$ & Groove \\
\hline Average & 23.81 & 24.18 & 16.10 & 22.72 & 22.03 & 24.12 & 21.69 & 25.00 & 15.98 & 18.88 & 10.45 & 12.09 \\
\hline $\begin{array}{l}\text { Standard } \\
\text { deviation }\end{array}$ & 7.98 & 3.51 & 4.67 & 6.08 & 6.97 & 2.93 & 5.14 & 6.99 & 5.37 & 2.45 & 5.19 & 1.95 \\
\hline
\end{tabular}

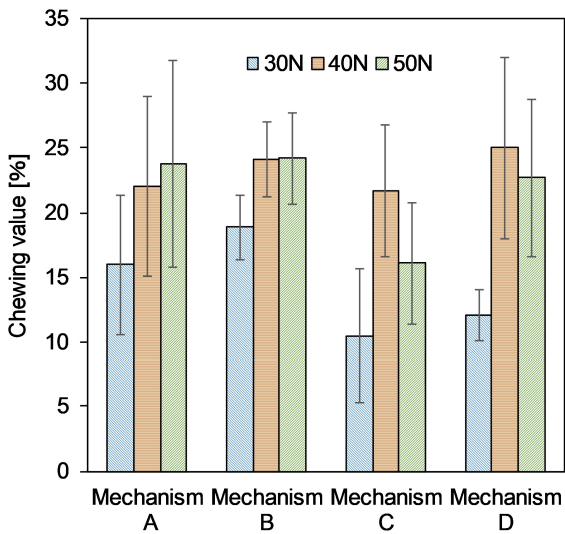

Fig. 5. Chewing value by Mechanism

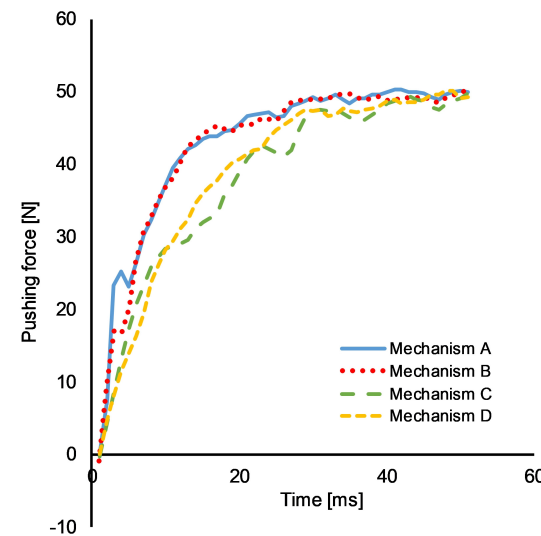

Fig. 6. Measured waveform with $50 \mathrm{~N}$ pushing force

To identify the factors associated with the lower chewing values in the mechanisms $\mathrm{C}$ and $\mathrm{D}$ with rotation, the measurement waves formed at less than $50 \mathrm{~N}$ pushing force were captured and analyzed. Fig. 6 shows the rise in the waveform for the mechanisms A through D. The rise in the waveform was more gradual for mechanisms $\mathrm{C}$ and $\mathrm{D}$ with springs, when compared to those for mechanisms A and B without springs. The results suggested that the rate of transmission of the pushing force decreased due to the springs. When the area under the curve (integral) of pushing force over time was calculated, the value was observed to be 9-12\% lower for the C-D group when compared to the A-B group (the time taken to reach the set value was taken as $50 \mathrm{~ms}$ ). These observations suggested that it was likely that the impact was lower in $\mathrm{C}$ and $\mathrm{D}$ mechanisms when compared to the A and B mechanisms, thereby decreasing the effect of grinding due to impact and subsequently leading to the lower chewing values that were observed. 


\section{Study of driving actuators}

\subsection{Motor-driven prototype model}

The results of various experiments with the chewing assistance mechanism indicated that the improvement in chewing ability was affected by the compressive force along with the grinding effect due to rotation. Also, the possible applications of ultrasonic motors as actuators for the powered robotic denture was investigated.

A small ultrasonic motor was used to realize the vertical and rotational movements around the lower and upper axes (Fig. 7. small TR motor) [4]. Miniaturization is more effective this way because of the simple mechanism where the shaft (rotor) is inserted into a hole bored through a dice-shaped metal (stator). A piezoelectric element is attached to the surface of the stator. Upon applying a voltage in the ultrasonic range to this area, an ultrasonic progression wave is generated on the inner surface of the stator hole, thereby enabling axial rotation and translation of the shaft.

As shown in Fig. 8, incorporating the mechanism of vertical movement in the inner part of a molar tooth will enable chewing in an occlusal condition.

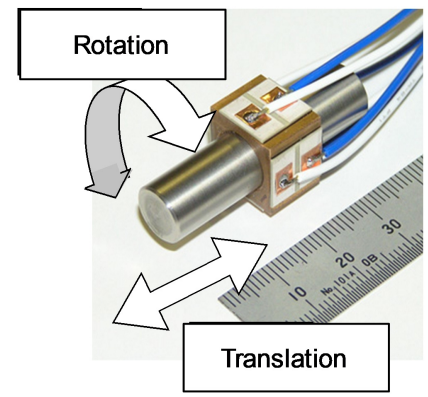

Fig. 7. TR motor (Translation-Rotation motor)

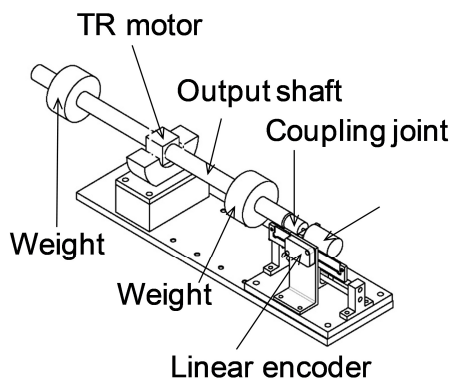

a)

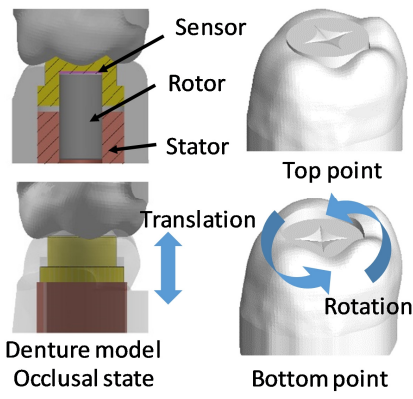

Fig. 8. Molar tooth model occlusal condition

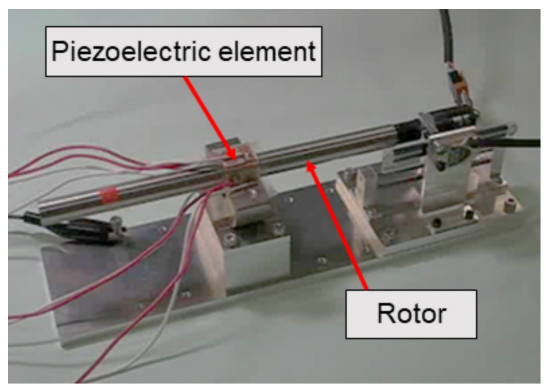

b)

Fig. 9. Driving experiment equipment

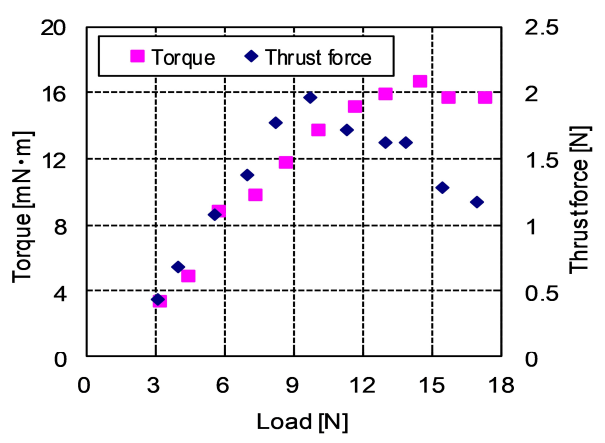

Fig. 10. Preload-output Characteristics

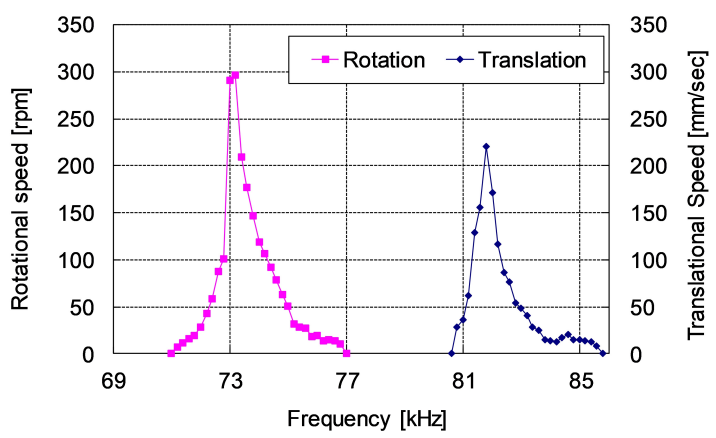

Fig. 11. Frequency characteristics (Preload 9.8 N) 


\subsection{TR motor driving experiments and discussions}

An experimental setup (shown in Fig. 9) was created to measure the output and frequency characteristics of the motor. The torque and thrust force were measured by using a thread and weight hung from a shaft shown in the figure. In the TR motor used in the experiments, phosphor bronze (C5191) was used as stator material and stainless steel (SUS303) was used as rotor material. The stator was shaped in the form of a $14 \mathrm{~mm}$ cube with a $10 \mathrm{~mm}$ hole bored through it. The shaft (rotor) was $150 \mathrm{~mm}$ long and weighed $92.3 \mathrm{~g}$. The inner and outer diameters of the stator were $10.009 \mathrm{~mm}$ and $9.998 \mathrm{~mm}$, respectively. The texture of the contact surface had an average roughness $3.2 \mu \mathrm{m}$. The impedance characteristics in a range of $60-90 \mathrm{kHz}$ were measured using an impedance analyzer.

Fig. 10 shows the preload-output characteristics of rotation and thrust force in the direction of translation. The maximum torque was $16.7 \mathrm{mN}$ with a preload of $14.5 \mathrm{~N}$ in the direction of rotation, and the maximum thrust was $1.96 \mathrm{~N}$ with a preload of $9.8 \mathrm{~N}$ in the direction of translation. Based on these results, the optimum preload was set at 9.8 N. Fig. 11 shows the frequency characteristics at the optimum preload. For both rotation and translation, the results showed a slight change in speed at a frequency band higher than the frequency at which maximum speed is obtained. This indicated that a frequency band higher than the resonance point is suitable for speed control.

\section{Conclusions}

This study involved the design, prototyping, and evaluation of a powered robotic denture for assisting elderly people without any disabilities with chewing. Using the developed prototype, it was possible to obtain the desired shape based on the design specification, and the results of the force of rotation and spring preload concurred with theoretical values. Based on experiments on the chewing value, it was discovered that the chewing value tended to be lower for mechanisms without any preprocessing (no grooves) on the occlusal surface and where only compression was utilized.

A TR motor was used as a driving actuator for the powered robotic denture, for which the optimum preload was investigated, and corresponding frequency characteristics were measured. Evaluations of the frequency characteristics at the optimum preload of $9.8 \mathrm{~N}$ revealed that a frequency band higher than the resonance point is suitable for speed control.

A potential topic for future research would be the selection of a suitable sieve to verify the effect of grinding due to friction and conducting further tests to evaluate the performance of the chewing assistance mechanism, along with the development of a TR motor actuator driver and driver program.

\section{Acknowledgements}

This work was supported by JSPS KAKENHI Grant Number JP18K04075.

\section{References}

[1] Takanobu T., Yajima T., Takanishi A., Otsuki K., Onishi M. Development of mouth-opening training robot using dental robotics technology and its application in therapy. Journal of the Japanese Society for the Temporomandibular Joint, Vol. 9, Issue 1, 1997, p. 72-79.

[2] Hashimoto Y., Sugimoto K., Oki K., Minagi S. Development of artificial tooth morphology having high masticatory efficiency with the purpose of reducing denture pain and diversifying chewable foods. Journal of the Japan Prosthodontic Society, Vol. 6, Issue 123, 2014, p. 195.

[3] Manly R. S., Braley L. C. Masticatory performance and efficiency. Journal of Dental Research, Vol. 29, Issue 4, 1950, p. 448-462.

[4] Nishizawa U., Toyama S. Phase difference control system for TR motor. Applied Mechanics and Materials, Vol. 841, 2016, p. 173-178. 